\title{
Genetic analysis of haptoglobin polymorphisms with cardiovascular disease and type 2 diabetes in the diabetes heart study
}

Jeremy N Adams ${ }^{1,2,3}$, Amanda J Cox ${ }^{2,3,4}$, Barry I Freedman ${ }^{5}$, Carl D Langefeld ${ }^{6}$, J Jeffrey Carr ${ }^{7}$ and Donald W Bowden $2,3,4^{*}$

\begin{abstract}
Background: Haptoglobin (HP) is an acute phase protein that binds to freely circulating hemoglobin. HP exists as two distinct forms, HP1 and HP2. The longer HP2 form has been associated with cardiovascular (CVD) events and mortality in individuals with type 2 diabetes (T2DM).

Methods: This study examined the association of HP genotypes with subclinical CVD, T2DM risk, and associated risk factors in a T2DM-enriched sample. Haptoglobin genotypes were determined in 1208 European Americans (EA) from 473 Diabetes Heart Study (DHS) families via PCR. Three promoter SNPs (rs5467, rs5470, and rs5471) were also genotyped.

Results: Analyses revealed association between HP2-2 duplication and increased carotid intima-media thickness (IMT; $\mathrm{p}=0.001)$. No association between HP and measures of calcified arterial plaque were observed, but the HP polymorphism was associated with triglyceride concentrations $(p=0.005)$ and CVD mortality $(p=0.04)$. We found that the HP2-2 genotype was associated with increased T2DM risk with an odds ratio (OR) of 1.49 (95\% Cl 1.18-1.86, $\left.p=6.59 \times 10^{-4}\right)$. Promoter SNPs were not associated with any traits.

Conclusions: This study suggests association between the HP duplication and IMT, triglycerides, CVD mortality, and T2DM in an EA population enriched for T2DM. Lack of association with atherosclerotic calcified plaque likely reflect differences in the pathogenesis of these CVD phenotypes. HP variation may contribute to the heritable risk for CVD complications in T2DM.
\end{abstract}

Keywords: Haptoglobin, Genetic polymorphism, Cardiovascular disease, Type 2 diabetes

\section{Introduction}

Cardiovascular disease (CVD) is one of the major complications associated with type 2 diabetes mellitus (T2DM). As of 2011, 25.8 million Americans had diagnosed T2DM [1]. More than $50 \%$ of individuals with T2DM had coronary heart disease, stroke, or cardiac disease [2]. T2DM is an independent risk factor for development of CVD with the relative risk of CVD mortality of 2.1 in men and 4.9 in women, relative to non-T2DM affected individuals $[3,4]$.

\footnotetext{
* Correspondence: dbowden@wakehealth.edu

${ }^{2}$ Center for Genomics and Personalized Medicine Research, Wake Forest

School of Medicine, Winston-Salem, North Carolina 27157, USA

${ }^{3}$ Center for Diabetes Research, Wake Forest School of Medicine,

Winston-Salem, North Carolina 27157, USA

Full list of author information is available at the end of the article
}

There is increasing evidence that genetic and environmental factors contribute to this risk.

Haptoglobin (HP) is a $54 \mathrm{kDa}$ protein, found abundantly in the serum $[5,6]$. The $H P$ gene has two major alleles: $H P 1$, (containing five exons) and $H P 2$, (containing seven exons) which likely arose from a duplication event involving exons 3 and 4, producing a $61 \mathrm{kDa}$ protein [6]. In its ancestral form, $\mathrm{HP}$ is a dimer, however, the $H P$ 1-2 encoded protein exists as linear polymers containing $2-8$ monomers, while the HP 2-2 encoded protein exists as circular polymers of 3-10 Hp monomers [6]. The expanded polymerization in the HP 1-2 and HP 2-2 genotypes is due to the duplication of the multimerization domain in exon 3 [6]. Genotype frequencies vary in different 
ethnicities. In European Americans (EA) they have been reported as 16\% HP 1-1, 48\% HP 1-2, and 36\% HP 2-2 [5].

HP may prevent oxidative damage through mechanisms including stabilization of the heme iron within hemoglobin (Hb) [7]. The HP-Hb complex is rapidly removed from circulation via CD163 mediated endocytosis by hepatic Kupfer cells [8]. The HP 1-1 protein is both more efficient than HP 2-2 at preventing oxidation caused by the heme iron [9] and is internalized and cleared from circulation more rapidly; with half lives of approximately 20 minutes and 50 minutes for $\mathrm{HP} 1-1-\mathrm{Hb}$ and $\mathrm{HP} 2-2-\mathrm{Hb}$ respectively $[10,11]$. Binding of the HP-Hb complex to CD163 induces the production of several cytokines and anti-inflammatory mediators $[9,12]$ with a much larger production of antiinflammatory mediators induced by HP 1-1 compared to HP 2-2 [13,14].

$\mathrm{HP}$ has been implicated in both T2DM and T2DMassociated CVD $[15,16]$. In the latter context the binding of $\mathrm{HP}$ to apolipoprotein A1 (ApoA1) has also been reported [17]. HP binds to ApoA1 in the same location as lecithin-cholesterol acyltransferase (LCAT), subsequently decreasing LCAT activity and therefore limiting high density lipoprotein (HDL) maturation. This inhibits reverse cholesterol transport causing HDL to become proatherogenic [17]. In addition, the tethering of $\mathrm{Hb}$ to $\mathrm{HDL}$ via the HP-ApoA1 allows the oxidation of HDL and its acquisition of proatherogenic and proinflammatory properties [18]. Due to the multimerization of the $\mathrm{Hp} 2$ protein, individuals with the $H P$ 2-2 genotype have significantly more $\mathrm{HP}$ attached to HDL via ApoA1 increasing these properties [11].

Due to the striking differences in properties of the HP 1 and HP 2 proteins, several studies have investigated the impact of the HP phenotype on CVD risk. There have been differing results when examining different populations and different outcomes. Studies investigating incident CVD in individuals affected by T2DM show an increased risk with the HP 2-2. One study [19] found that individuals with T2DM and the HP2-2 genotype had increased risk for CVD events. In addition, Suleiman et al. in 2005 [20] found that individuals with T2DM and the HP 1-1 phenotype had decreased 30-day mortality and heart failure after acute myocardial infarction compared to individuals with the HP 2-2 phenotype, again suggesting the HP 2-2 phenotype as the risk phenotype. This association was not seen in individuals without T2DM. Indeed similar observations have been made in type 1 diabetes; Simpson et al. [21] found that in individuals with type 1 diabetes the HP 2-2 genotype predicted coronary artery calcification progression, a measure of subclinical CVD. In contrast, in cohorts where rates of T2DM are low or individuals with T2DM excluded, the HP1-1 genotype has been shown to be associated with an increased risk for mortality due to coronary heart disease
[22]. Similarly, in the Framingham offspring study the HP 1-2 or HP 2-2 phenotypes were associated with decreased rates of prevalent CHD [23].

The HP duplication has also been examined for association with T2DM. The role of HP in regulation of inflammation suggests a potential role in T2DM pathogenesis. There are several studies showing that the HP duplication was associated with T2DM risk in different populations [24,25].

Thus, the relationship between $H P$ polymorphism and CVD in T2DM-affected individuals is likely complex and association with T2D risk has been documented to a limited degree. Based on these prior studies we hypothesized that if the HP 2-2 genotype is associated with CVD events in people with T2DM, then a similar association would likely be observed with measures of subclinical CVD in predominately T2DM-affected populations. We have taken advantage of the richly phenotyped Diabetes Heart Study [26] (DHS) sample with measures of coronary artery calcification (CAC; or calcified plaque), carotid wall intima-medial thickness (IMT), and blood lipid traits to investigate this hypothesis. Further, the DHS provides a base from which to investigate whether the HP locus is directly associated with T2DM risk.

\section{Methods}

\section{Subjects}

The DHS is a study of the genetic and epidemiological causes of CVD in individuals with T2DM. Ascertainment, recruitment, and examination have been previously described in detail [26]. Briefly, siblings concordant for T2DM and without serious health conditions, e.g. renal replacement therapy, were recruited. T2DM was defined as diabetes developing after 35 years of age, treatment with insulin and/or oral agents and absence of historical evidence of ketoacidosis. If available, additional non-T2DM affected siblings were recruited simultaneously using criteria above to exclude T2DM. The 1208 DHS EA individuals used in this analysis were from 473 families.

\section{Clinical evaluation}

The protocols for this study were approved by the Institutional Review Board at Wake Forest School of Medicine; written informed consent was received prior to participation. Examinations were conducted in the General Clinical Research Center of the Wake Forest Baptist Medical Center, and included interviews for medical history and health behaviors, anthropometric measures, resting blood pressure, electrocardiography, fasting blood sampling and spot urine collection. Individuals reported history of prior CVD based on prior event (angina, myocardial infarction, stroke) and/or intervention (coronary angiography, coronary artery bypass grafting, carotid endarterectomy). CAC, carotid artery calcified plaque ( $\mathrm{CarCP}$ ) and infra-renal abdominal 
aortic calcified plaque (AACP) were measured using fastgated helical CT scanning, and calcium scores calculated as previously described and reported as an Agatston score $[27,28]$. Carotid IMT was measured by high-resolution Bmode ultrasonography with a $7.5-\mathrm{MHz}$ transducer and a Biosound Esaote (AU5) ultrasound machine (Biosound Esaote, Inc., Indianapolis, IN) as previously described [29]. All measurements were not available for all participants.

\section{Mortality}

Vital status was determined from the National Social Security Death Index maintained by the United States Social Security Administration. For participants confirmed as deceased, length of follow-up was determined from data of the initial study visit to date of death. For deceased participants, copies of death certificates were obtained from relevant county Vital Records Offices to confirm cause of death. For all other participants the length of follow-up was determined from the date of the initial study visit to January 1, 2011. Cause of death was categorized based on information contained in death certificates as CVD-related (myocardial infarction, congestive heart failure, cardiac arrhythmia, sudden cardiac death, peripheral vascular disease, and stroke) or cancer, infection, end-stage renal disease, accidental, or other (including obstructive pulmonary disease, pulmonary fibrosis, liver failure and Alzheimer's dementia).

\section{Genotyping}

Genomic DNA was purified from whole-blood samples obtained from subjects using the PUREGENE DNA isolation kit (Gentra Systems., Minneapolis, MN). DNA was quantitated using standardized fluorometric readings on a Hoefer DyNA Quant 200 fluorometer (Hoefer Pharmacia Biotech, Inc., San Francisco, CA). Each sample was diluted to a final concentration of $20 \mathrm{ng} / \mu \mathrm{L}$. HP duplication genotyping was performed using paired polymerase chain reactions (PCR). PCR primers, reaction and cycling conditions were performed as described previously by Koch et al. [30] for $10 \mu \mathrm{L}$ reactions containing 45-60 ng DNA and primers at final concentrations of $0.8 \mu \mathrm{M}$ for the $H P 1$ and $0.6 \mu \mathrm{M}$ for the HP2.

PCR products from the two reactions were combined $(10 \mu \mathrm{L}$ of the HP1 product with $5 \mu \mathrm{L}$ of the HP2 PCR product) and resolved on a $1 \%$ agarose gel (Figure 1) which was visualized by staining with $1.4 \%$ ethidium bromide and the images captured using an Alpha Imager (Alpha Innotech, San Leandro, CA). Haptoglobin genotypes were called independently by two investigators (JNA and AJC) with $100 \%$ concordance between calls. Genotyping also included a total of 29 blind duplicates to allow for evaluation of genotyping accuracy. The concordance rate for these blind duplicates was $100 \%$.

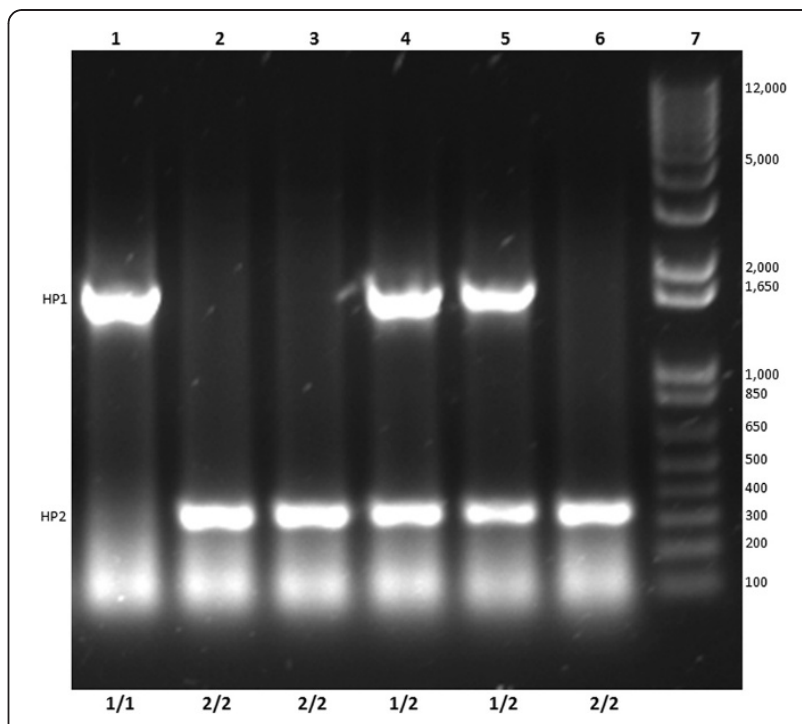

Figure $1 \mathrm{Gel}$ picture of HP PCR products. Example of HP genotype discrimination using PCR reaction products resolved on a $1.4 \%$ agarose gel with ethidium bromide staining where lanes 1-6 are participant samples and lane 7 a DNA MW size ladder. Lane numbers are at the top and genotyping call is at the bottom.

\section{HP promoter polymorphism genotyping}

In addition to the classical HP1 and HP2 variants, three single nucleotide polymorphisms (SNPs), rs5467, rs5470, and rs5471, located in the promoter region of HP and previously reported to be associated with circulating $\mathrm{HP}$, were genotyped in the DHS. Genotyping was performed using the Sequenom Mass ARRAY genotyping system (Sequenom, San Diego, CA) and PCR primers were designed using the Mass ARRAY Assay Design 3.4 Software (Sequenom). These SNPs are in low linkage disequilibrium (LD) with each other or with the HP duplication $\left(r^{2} \leq 0.03\right)$ (see Additional file 1). An additional 41 quality control samples were included in the genotyping analysis to serve as blind duplicates. The concordance rate for these blind duplicates was $100 \%$. The minimum acceptable call frequency for all SNPs was 95\%. The average call frequency was $98.4 \pm 0.001 \%$ (mean \pm SD). Samples with genotyping efficiency rates $<90 \%$ were excluded from further analysis.

T2DM-affected cases and controls for analysis of T2DM risk An additional 606 EA T2DM cases and 985 EA non-T2DM affected controls from an independent study of EA T2DM [31,32] were genotyped for the HP duplication using the PCR method described above. Thirty-one additional quality control samples were included as blind duplicates. The concordance rate for these samples was $100 \%$. For analysis we combined HP data for unrelated T2DM cases from DHS $(n=473)$ with T2DM cases from the independent T2DM case sample for a total of 1079 cases. These data were evaluated for association with $H P$ genotypes contrasting with controls without T2DM $(\mathrm{n}=985)$. 


\section{Statistical analysis}

$H P$ allele frequencies were calculated for a sub-set of unrelated individuals and departure from Hardy-Weinberg equilibrium was calculated from a group of unrelated samples using a chi-squared goodness-of-fit test. Association between the HP genotypes and subclinical CVD measures was examined using variance component methods computed using SOLAR v4.3.1 (Texas Biomedical Research Institute, San Antonio, TX, USA). Each trait was examined using additive, dominant, and recessive models of inheritance. Continuous variables were transformed prior to analysis to approximate conditional normality. Age, gender, and T2DM-affection status were used as covariates in analysis of quantitative traits. Additional covariates (smoking, hypertension, C-reactive protein, lipid medication use, hypertension medication use, and T2DM duration) were also evaluated, but did not impact the results. Analyses were repeated in the subset of the population that was affected by T2DM. Statistical significance was accepted at $\mathrm{p}<0.05$. The program SNPGWA (http://www.phs.wfubmc. edu/public_bios/sec_gene/downloads.cfm) [33] was used to test for associations between HP and T2DM risk comparing individuals affected with T2DM (DHS T2DM-affected unrelated individuals $[n=473]$ and EA T2DM-affected individuals $[n=606])$ to the EA non-T2DM affected controls $(n=985)$. Covariates used in this analysis were age, gender, and body mass index (BMI). Analyses included samples that had genotype and all covariate data.

\section{Results}

Characteristics of the DHS sample are summarized in Table 1 including the mean trait values for the overall sample and for each HP genotype group. Briefly, the mean age of the sample was 61.5 years; 1013 individuals (83.86\%) were T2DM-affected and slightly more than 50\% (643) were female. Overall, the characteristics of the DHS

Table 1 Demographic characteristics of DHS samples, including HP duplication genotypes (data shown are mean \pm SD, unless specified otherwise)

\begin{tabular}{|c|c|c|c|c|}
\hline \multicolumn{5}{|c|}{ DHS Demographics } \\
\hline & \multirow[b]{2}{*}{ Total } & \multicolumn{3}{|c|}{ HP Genotypes } \\
\hline & & $1 / 1$ & $1 / 2$ & $2 / 2$ \\
\hline Number & 1208 & 168 & 505 & 535 \\
\hline Age (years) & $61.5 \pm 9.35$ & $62.5 \pm 9.36$ & $61.2 \pm 9.34$ & $61.5 \pm 9.36$ \\
\hline Female (\%) & $643(53.2)$ & $87(51.8)$ & $274(54.3)$ & $282(52.7)$ \\
\hline BMI $\left(\mathrm{kg} / \mathrm{m}^{2}\right)$ & $31.8 \pm 6.49$ & $31.9 \pm 6.27$ & $32.1 \pm 6.81$ & $31.4 \pm 6.23$ \\
\hline Affected (\%) & $1013(83.9)$ & $134(79.8)$ & $431(85.4)$ & $448(83.7)$ \\
\hline Diabetes duration (years) & $10.41 \pm 7.1$ & $10.95 \pm 7.9$ & $10.15 \pm 7.24$ & $10.49 \pm 6.86$ \\
\hline Metabolic Syndrome (\%) & $1029(85.2)$ & $140(83.3)$ & $434(85.9)$ & $455(85.1)$ \\
\hline Glucose (mg/dL) & $139.4 \pm 55.5$ & $139.7 \pm 58.8$ & $142.3 \pm 56.7$ & $136.7 \pm 53.1$ \\
\hline HbA1c (\%) & $7.29 \pm 1.7$ & $7.13 \pm 1.57$ & $7.31 \pm 1.78$ & $7.32 \pm 1.78$ \\
\hline C-reactive protein (mg/dl) & $0.59 \pm 0.97$ & $0.54 \pm 0.81$ & $0.60 \pm 1.05$ & $0.59 \pm 0.94$ \\
\hline Hypertension (\%) & $1025(84.85)$ & $141(83.93)$ & $422(83.56)$ & $462(86.36)$ \\
\hline Smoking (\%) & $708(58.85)$ & $96(57.49)$ & $285(56.55)$ & $327(61.47)$ \\
\hline Lipid medication (\%) & $540(44.7)$ & $76(45.24)$ & $221(43.76)$ & $243(45.42)$ \\
\hline Anti-hypertensive medication (\%) & $747(61.84)$ & $101(60.12)$ & $324(64.16)$ & $322(60.19)$ \\
\hline CAC & $1662.5 \pm 3160.7$ & $1679.1 \pm 2449.8$ & $1607.2 \pm 3001.5$ & $1708.5 \pm 3492.3$ \\
\hline CarCP & $312.9 \pm 672.1$ & $276.2 \pm 522.5$ & $292.8 \pm 610.8$ & $343.3 \pm 762.4$ \\
\hline AACP & $10949.3 \pm 15748.8$ & $12628.86 \pm 16231.1$ & $9781.9 \pm 14236.0$ & $11458.1 \pm 16775.3$ \\
\hline IMT (mm) & $0.676 \pm 0.134$ & $0.677 \pm 0.136$ & $0.665 \pm 0.129$ & $0.686 \pm 0.139$ \\
\hline Cholesterol (mg/dL) & $186.8 \pm 42.4$ & $187.5 \pm 46.2$ & $187.7 \pm 43.4$ & $185.8 \pm 40.3$ \\
\hline $\mathrm{LDL}(\mathrm{mg} / \mathrm{dL})$ & $105.1 \pm 32.7$ & $104.0 \pm 35.3$ & $103.3 \pm 30.8$ & $107.1 \pm 33.5$ \\
\hline $\mathrm{HDL}(\mathrm{mg} / \mathrm{dL})$ & $43.1 \pm 12.5$ & $43.5 \pm 14.4$ & $43.3 \pm 12.7$ & $42.7 \pm 11.6$ \\
\hline Triglycerides (mg/dL) & $201.4 \pm 132.1$ & $212.8 \pm 162.3$ & $208.8 \pm 133.7$ & $190.9 \pm 119.2$ \\
\hline History of CVD (\%) & $471(38.99)$ & $72(42.86)$ & $191(37.82)$ & $208(38.88)$ \\
\hline Non-CVD Mortality (\%) & $122(10.10)$ & $19(11.31)$ & $49(9.70)$ & $54(10.09)$ \\
\hline CVD Mortality (\%) & $100(8.28)$ & $11(6.55)$ & $34(6.73)$ & $55(10.28)$ \\
\hline
\end{tabular}


sample are representative of T2DM-affected patients in the general population.

HP duplication genotypes were determined for 1208 individuals (Table 1). The genotype frequencies were 13.9\% HP 1-1, 41.8\% HP 1-2, and 44.3\% HP 2-2 and were consistent with Hardy-Weinberg proportions.

\section{CVD associations}

HP duplication data was analyzed for association with multiple CVD measures, risk factors, and outcomes: calcified plaque (CAC, CarCP, and AACP), carotid IMT, lipids, prevalent CVD and mortality. The $H P$ duplication was associated with carotid IMT $(\mathrm{p}=0.001$, Table 2$)$. We did not, however, observe a significant association of HP with measures of vascular calcification: CAC, CarCP or AACP (Table 2). There did not appear to be any discernable trend of increased calcification in the mean trait values by genotype for any of the three arterial beds (Table 1). Additional analyses performed in T2DM-affected individuals alone, revealed similar results (data not shown).

A total of 471 (39.0\%) individuals in the DHS have a self reported history of prior CVD. This included 72 individuals (42.9\%) for HP 1-1, for HP 1-2 genotype 191 (37.8\%), and for HP 2-2 208 (38.98\%). However, prevalent CVD was not associated with the HP duplication ( $\mathrm{p}=0.23-0.94$; Table 2$)$.

The HP2 allele was associated with serum triglyceride concentrations $(\mathrm{p}=0.005$; Table 2$)$. With each additional copy of the HP2 allele, triglyceride concentrations decreased and were on average $10.3 \mathrm{mg} / \mathrm{dL}$ lower with the HP2-2 genotype (Table 1). In addition, the HP2 allele was not associated with all cause mortality $(\mathrm{p}=0.17)$; however, it was nominally associated with CVD mortality $(\mathrm{p}=0.04$; Table 2). Results were essentially unchanged when analyses were repeated in T2DM affected individuals only (see Additional file 2).

\section{HP promoter polymorphisms}

SNPs rs5467, rs5470, and rs5471 were genotyped based on prior reports that they were associated with HP protein concentrations $[34,35]$. Each SNP was in Hardy-Weinberg equilibrium. Minor allele frequencies (MAF) were 19.2\% for rs5467, $0.04 \%$ for rs5470, and $0.08 \%$ for rs 5471 . None of these HP SNPs were associated with any of the subclinical CVD traits, CVD risk factors, events, or mortality ascertained in the current study (see Additional file 3).

\section{HP polymorphism and T2DM risk}

Table 3 contains the clinical demographics of the T2DM case and control samples. This sample was ascertained and recruited independently from the DHS, but using the same diagnostic criteria for T2DM diagnosis and has been the basis for prior genetic studies of T2DM [31,32]. Briefly, the mean age of the T2DM-affected case sample was $65.2 \pm 9.9$ years compared to $53.8 \pm 15.0$ for the nonT2DM controls. Fewer than $50 \%(\mathrm{n}=299)$ of cases and greater than $63 \%(n=628)$ of the controls were female.

For all T2DM cases, genotype frequencies were $14.1 \%$ HP 1-1, 43.4\% HP 1-2, and 42.5\% HP 2-2. In non-T2DM controls, genotype frequencies were $15.8 \%$ HP 1-1, 50.1\% HP 1-2, and 34.1\% HP 2-2 (Table 3). Genotype frequencies in both samples were consistent with Hardy-Weinberg proportions. Individuals with the HP 2-2 genotype were found to be more likely to have T2DM (recessive model OR: $1.49 ; 95 \%$ CI: $\left.1.18-1.86 ; \mathrm{p}=6.59 \times 10^{-4}\right)$. Similar to the DHS analysis of CVD, there was no evidence of association of T2DM risk with HP promoter SNPs in the DHS (data not shown).

\section{Discussion}

This study evaluated association of HP gene polymorphisms with subclinical CVD, mortality, and T2DM in 1208 EA individuals from the DHS. The HP 2-2 genotype was

Table 2 Association result for the HP duplication with measures of subclinical CVD and blood lipids

\begin{tabular}{|c|c|c|c|c|c|c|c|}
\hline \multicolumn{8}{|c|}{ HP Duplication - CVD Association Results } \\
\hline & \multirow[b]{2}{*}{$\mathbf{N}$} & \multicolumn{2}{|c|}{ Additive } & \multicolumn{2}{|c|}{ Dominant } & \multicolumn{2}{|c|}{ Recessive } \\
\hline & & Beta \pm SE & $\overline{p \text {-value }}$ & Beta \pm SE & $\overline{p \text {-value }}$ & Beta \pm SE & $\overline{p \text {-value }}$ \\
\hline$\overline{C A C}$ & 1134 & $0.022 \pm 0.097$ & 0.82 & $-0.025 \pm 0.135$ & 0.85 & $0.135 \pm 0.189$ & 0.48 \\
\hline CarCP & 1142 & $-0.159 \pm 0.102$ & 0.12 & $-0.180 \pm 0.142$ & 0.21 & $-0.255 \pm 0.200$ & 0.20 \\
\hline AACP & 865 & $-0.025 \pm 0.102$ & 0.81 & $-0.117 \pm 0.141$ & 0.41 & $0.141 \pm 0.202$ & 0.48 \\
\hline IMT & 1103 & $-0.008 \pm 0.003$ & 0.01 & $-0.014 \pm 0.004$ & 0.001 & $-0.002 \pm 0.006$ & 0.79 \\
\hline Cholesterol & 1188 & $0.006 \pm 0.010$ & 0.53 & $0.014 \pm 0.013$ & 0.28 & $-0.005 \pm 0.019$ & 0.78 \\
\hline Triglycerides & 1188 & $0.060 \pm 0.024$ & 0.01 & $0.091 \pm 0.033$ & 0.005 & $0.050 \pm 0.047$ & 0.28 \\
\hline Prevalent CVD & 1084 & $-0.033 \pm 0.061$ & 0.58 & $0.006 \pm 0.083$ & 0.94 & $-0.140 \pm 0.117$ & 0.23 \\
\hline All Cause Mortality & 1208 & $0.063 \pm 0.065$ & 0.33 & $0.123 \pm 0.089$ & 0.17 & $-0.004 \pm 0.129$ & 0.98 \\
\hline CVD Mortality & 1208 & $0.145 \pm 0.081$ & 0.07 & $0.222 \pm 0.101$ & 0.04 & $0.117 \pm 0.116$ & 0.47 \\
\hline
\end{tabular}

Associations were examined under additive, dominant and recessive genetic models. Bold indicates statistical significance. CAC: coronary artery calcified plaque; CarCP: carotid artery calcified plaque; AACP: abdominal aortic calcified plaque; IMT: carotid intima-media thickness. SE = standard error. 
Table 3 Demographic characteristics of T2DM case and non-diabetic control samples, including HP duplication genotype measures (data shown is mean \pm SD unless specified otherwise)

\begin{tabular}{llllll}
\hline & \multicolumn{5}{c}{ T2DM Demographics } \\
\hline & T2DM Cases & DHS T2DM Cases & Total T2DM Cases & Non-diabetes controls & Total \\
\hline Number & 606 & 473 & 1079 & 985 & 2064 \\
Age (years) & $65.18 \pm 10.45$ & $62.09 \pm 8.86$ & $63.79 \pm 9.88$ & $53.83 \pm 15.03$ & $59.82 \pm 13.13$ \\
Female (\%) & $299(49.42)$ & $246(52.01)$ & $545(50.06)$ & $628(63.82)$ & $1173(56.89)$ \\
BMI (kg/m2) & $29.65 \pm 7.15$ & $32.64 \pm 6.53$ & $30.97 \pm 7.04$ & $28.35 \pm 5.67$ & $29.97 \pm 6.67$ \\
Affected (\%) & $606(100)$ & $473(100)$ & $1079(100)$ & $0(0.00)$ & $1079(52.13)$ \\
$1 / 1$ Genotype (\%) & $88(14.52)$ & $64(13.53)$ & $152(14.09)$ & $156(15.84)$ & $308(14.92)$ \\
$1 / 2$ Genotype (\%) & $274(45.21)$ & $194(41.01)$ & $468(43.37)$ & $493(50.05)$ & $361(46.66)$ \\
2/2 Genotype (\%) & $244(40.26)$ & $215(45.45)$ & $459(42.53)$ & $336(34.11)$ & $795(38.52)$ \\
\hline
\end{tabular}

associated with increased carotid IMT $(\mathrm{p}=0.001$, Table 1$)$ in this T2DM enriched population. However, we did not observe significant evidence of association between $H P$ genotype and calcified plaque as a different measure of subclinical CVD. An association with triglyceride concentrations was also observed; the HP 2-2 genotype was associated with lower concentrations. The biological mechanism for this latter association is as of yet, unknown. We also observed suggestive evidence for association of the $H P$ duplication polymorphism with CVD related mortality in the DHS. In addition, we found that the HP 2-2 genotype was associated with T2DM status (OR: 1.49; 95\% CI: 1.18$1.86 ; \mathrm{p}=6.59 \times 10^{-4}$ ).

Several prior studies have investigated $H P$ polymorphisms and CVD risk in T2DM. In 2002 Levy et al. [19] reported an OR of CVD events in diabetes five times greater with the HP 2-2 phenotype, than with HP 1-1 in a study that included 206 CVD patients and 206 CVD controls (146 and 93 were affected by T2DM, respectively, as part of the Strong Heart Study). In 2004, a subsequent study by Levy et al. [23] included 3273 individuals in the Framingham Heart Study, however only a subset of 433 individuals were affected with T2DM, and of these, only 86 had a history of prevalent CVD. Finally, a 2003 study in individuals with acute myocardial infarction (AMI) reported individuals with T2DM and the HP2 allele had increased mortality following AMI compared to individuals with T2DM and the HP 1-1 genotype (included only 224 T2DMaffected individuals) [20]. In the present study we detected modest evidence of association with carotid IMT, but did not strongly replicate association with history of prior CVD and only nominally with CVD mortality. Parenthetically, IMT and measures of vascular calcification are not highly correlated [26]. The DHS is predominately comprised of T2DM-affected subjects (1013 of 1208 participants). Our primary measures were the subclinical measures of CVD, CAC and IMT which may not be as strongly influenced by $H P$ polymorphism. Of the DHS subjects, 435 were T2DM-affected participants with a history of prevalent CVD, based upon self-reported history and prior intervention which was not associated with $H P$ genotype. The analysis with CVD mortality, a firm endpoint, suggests a possible contribution to risk. Given the association of the HP 2-2 genotype with risk for mortality, it is possible that a survival bias may be present. However, genotype frequencies were consistent with Hardy-Weinberg equilibrium. In addition, the genotype frequencies of the HP duplication in this study were similar to those reported previously [5].

In prior reports, two promoter SNPs, rs5470 and rs5471 were associated with altered levels of $H P$ expression [34,35] with rs5471 reported to be associated with the Haptoglobin 1-2 modified (HP1-2mod) phenotype. In individuals with the rs5471 "C" allele and the HP 1-2 genotype, normal expression levels of the HP 1 protein, but decreased levels of HP 2 have been reported [34]. It has been suggested that the decreased levels of HP 2 lead to greater oxidative stress [36]. We did not observe evidence of association for these three genotyped HP SNPs genotyped (rs5467, rs5470, and rs5471) with measures of subclinical CVD, history of CVD, or mortality. One possible explanation is the low MAF for both rs5470, and rs5471 (0.0004 and 0.0008 respectively). The combination of the HP1-2 and the rs5471 SNP has been reported in approximately $10 \%$ of African Americans [34], but we are unaware of reports of the frequency of the HP1-2mod phenotype in other populations. In this study there were no minor allele homozygotes for rs5471, nor rs5471 heterozygotes with the HP1-2 genotype; as such, the Haptoglobin 1-2 modified phenotype is unlikely to have confounded the HP associations described here. In addition, in LD analysis (see Additional file 1) these two SNPs were in low LD with the HP duplication. Thus these two promoter SNPs along with rs5467 probably do not have an impact on CVD or T2DM status. However, there are other SNPs that are known to impact circulating HP concentrations (e.g. rs2000999) [37] that we did not genotype in the current study which may also contribute to the variance in $\mathrm{HP}$ 
and its role in CVD risk. The lack of measured HP concentrations in the DHS and the inability to further control for these additional genetic variants is one limitation of this work.

Several previous studies have investigated the effect of the HP polymorphism on T2DM risk. A previous study by Stern et al. in 1986 [24] found that the HP1 allele was associated diabetes risk in Mexican Americans. They found that a single copy of the HP1 allele increased T2DM risk by $50 \%$ and a second copy increased risk by $100 \%$. A second report from 2006 by Quaye et al. [25] found that the HP 2-2 phenotype was a risk factor for T2DM in a population in Ghana. The current study had a larger sample size than either of the two previous studies, albeit in a different ethnicity. In this study it was found that EA individuals with the $H P$ 2-2 genotype are more likely to have T2DM with an OR of 1.49. These studies, when combined suggest that $H P$ is a risk gene for diabetes or is in LD with a risk gene. Several studies have shown that the different alleles lead to different levels of circulating HP protein [22,37]. Higher circulating HP has been suggested to be associated with metabolic syndrome, high blood pressure, and elevated glucose [38]. This could possibly explain the association of the HP polymorphism with T2DM. Different risk alleles across populations are problematic as it could be difficult to assess risk across different populations.

Importantly, the T2DM association may be difficult to further investigate without subsequent data generation since the duplication is not included in the major, publically available, databases. Furthermore, any subsequent studies will require a targeted phenotyping approach either through analysis of HP in serum or genotyping through fragment size analysis as performed in this study, since the duplication is not captured by the current commercially available genome-wide genotyping platforms and does not appear to be tagged by other common polymorphisms [37]. A genome wide association study (GWAS) analysis has been performed in the DHS and LD was analyzed for the HP duplication and the two SNPs on the GWAS chip that are closest to the duplication (rs16973636 and rs2287998). The SNPs were found to have low LD with the duplication with an $\mathrm{r}^{2}$ of $\leq 0.01$ (data not shown).

\section{Conclusions}

Overall, we detected limited association of haptoglobin polymorphisms with CVD. The HP2-2 genotype was found to be positively associated with carotid IMT and the HP2 allele was associated with decreased serum triglyceride concentrations. We identified an association with T2DM in EAs that has not been reported previously [39-41]. Further studies are needed to extend and replicate these relationships.

\section{Additional files}

Additional file 1: LD of HP duplication and promoter SNPs. LD plot showing $r^{2}$ between the HP duplication (HPDup) and genotyped promoter SNPs (rs5467, rs5470, and rs5471) based on genotypes from the DHS sample.

Additional file 2: HP Duplication - T2DM CVD Association Results. Association results for the HP duplication in the individuals with T2DM only with measures of subclinical CVD (CAC: coronary artery calcified plaque; CarCP: carotid artery calcified plaque; AACP: abdominal aortic calcified plaque; IMT: carotid intima-media thickness) and blood lipids. Associations were examined under additive, dominant and recessive genetic models. Bold indicates statistical significance. SE = standard error.

Additional file 3: HP Promoter SNP - CVD Association Results. Association analysis of HP promoter SNPs with subclinical CVD (CAC: coronary artery calcified plaque; CarCP: carotid artery calcified plaque; AACP: abdominal aortic calcified plaque; IMT: carotid intima-media thickness) and blood lipids. Associations were examined under an additive model. $\mathrm{SE}=$ standard error.

\section{Abbreviations}

AACP: Infra-renal abdominal aortic calcified plaque; ApoA1: Apolipoprotein A1; BMl: Body mass index; CAC: Coronary artery calcified plaque; CarCP: Carotid artery calcified plaque; CVD: Cardiovascular disease; DHS: Diabetes Heart Study; EA: European American; GWAS: Genome wide association study; Hb: Hemoglobin; HDL: High density lipoprotein; HP: Haptoglobin; IMT: Intima media thickness; LCAT: Lecithin-cholesterol acyltransferase; LD: Linkage disequilibrium; MAF: Minor allele frequency; OR: Odds ratio; PCR: Polymerase chain reaction; SNP: Single nucleotide polymorphism; T2DM: Type 2 diabetes mellitus.

\section{Competing interests}

The authors declare they have no competing interests.

\section{Authors' contributions}

JNA perfomed the duplication genotyping and statistical analysis and wrote the manuscript; AJC performed the SNP genotyping, assisted with the duplication genotyping, and assisted with the manuscript preparation; BIF was involved in the conception of the DHS, participated in subject recruitment and clinical assessment and reviewed the manuscript; CLD contributed to statistical analyses and reviewed the manuscript; JJC was involved in the conception of the DHS, participated in subject recruitment and clinical assessment, and reviewed the manuscript; DWB designed and supervised the DHS, conceived the haptoglobin investigation and assisted with the manuscript preparation. All authors read and approved the final manuscript.

\section{Acknowledgements}

This study was supported by R01 HL67348, R01 HL092301, and R01 NS058700 to D.W.B.

\section{Author details}

${ }^{1}$ Molecular Genetics and Genomics Program, Wake Forest School of Medicine, Winston-Salem, North Carolina 27157, USA. ${ }^{2}$ Center for Genomics and Personalized Medicine Research, Wake Forest School of Medicine, Winston-Salem, North Carolina 27157, USA. ${ }^{3}$ Center for Diabetes Research, Wake Forest School of Medicine, Winston-Salem, North Carolina 27157, USA ${ }^{4}$ Department of Biochemistry, Wake Forest School of Medicine, Medical Center Boulevard, Winston-Salem, North Carolina 27157, USA. ${ }^{5}$ Department of Internal Medicine - Nephrology, Wake Forest School of Medicine, Winston-Salem, North Carolina 27157, USA. ${ }^{6}$ Division of Public Health Sciences, Department of Biostatistical Sciences, Wake Forest School of Medicine, Winston-Salem, North Carolina 27157, USA. ${ }^{7}$ Department of Radiologic Sciences, Wake Forest School of Medicine, Winston-Salem, North Carolina 27157, USA.

Received: 12 November 2012 Accepted: 1 February 2013 Published: 11 February 2013 


\section{References}

1. Centers for Disease Control and Prevention: National diabetes fact sheet: national estimates and general information on diabetes and prediabetes in the United States, 2011. Atlanta, GA: U.S. Department of Health and Human Services, Centers for Disease Control and Prevention; 2011.

2. Bowden DW, Cox AJ, Freedman Bl, Hugenschimdt CE, Wagenknecht LE, Herrington D, Agarwal S, Register TC, Maldjian JA, Ng MC, et al: Review of the diabetes heart study (DHS) family of studies: a comprehensively examined sample for genetic and epidemiological studies of type 2 diabetes and its complications. Rev Diabet Stud 2010, 7(3):188-201.

3. Diabetes mellitus: a major risk factor for cardiovascular disease. A joint editorial statement by the american diabetes association; the national heart, lung, and blood institute; the juvenile diabetes foundation international; the national institute of diabetes and digestive and kidney diseases; and the american heart association. Circulation 1999, 100(10): 1132-1133.

4. Kannel WB, McGee DL: Diabetes and cardiovascular disease. The Framingham study. JAMA 1979, 241(19):2035-2038.

5. Langlois MR, Delanghe JR: Biological and clinical significance of haptoglobin polymorphism in humans. Clin Chem 1996, 42(10):1589-1600.

6. Levy AP, Asleh R, Blum S, Levy NS, Miller-Lotan R, Kalet-Litman S, Anbinder Y, Lache O, Nakhoul FM, Asaf R, et al: Haptoglobin: basic and clinical aspects. Antioxid Redox Signal 2010, 12(2):293-304.

7. Bunn HF, Jandl JH: Exchange of heme among hemoglobin molecules. Proc Natl Acad Sci U S A 1966, 56(3):974-978.

8. Graversen JH, Madsen M, Moestrup SK: CD163: a signal receptor scavenging haptoglobin-hemoglobin complexes from plasma. Int $\mathrm{J}$ Biochem Cell Biol 2002, 34(4):309-314.

9. Schaer CA, Schoedon G, Imhof A, Kurrer MO, Schaer DJ: Constitutive endocytosis of CD163 mediates hemoglobin-heme uptake and determines the noninflammatory and protective transcriptional response of macrophages to hemoglobin. Circ Res 2006, 99(9):943-950.

10. Asleh R, Marsh S, Shilkrut M, Binah O, Guetta J, Lejbkowicz F, Enav B, Shehadeh N, Kanter Y, Lache O, et al: Genetically determined heterogeneity in hemoglobin scavenging and susceptibility to diabetic cardiovascular disease. Circ Res 2003, 92(11):1193-1200.

11. Asleh R, Blum S, Kalet-Litman S, Alshiek J, Miller-Lotan R, Asaf R, Rock W, Aviram M, Milman U, Shapira C, et al: Correction of HDL dysfunction in individuals with diabetes and the haptoglobin 2-2 genotype. Diabetes 2008, 57(10):2794-2800.

12. Melamed-Frank M, Lache O, Enav BI, Szafranek T, Levy NS, Ricklis RM, Levy AP: Structure-function analysis of the antioxidant properties of haptoglobin. Blood 2001, 98(13):3693-3698.

13. Guetta J, Strauss M, Levy NS, Fahoum L, Levy AP: Haptoglobin genotype modulates the balance of Th1/Th2 cytokines produced by macrophages exposed to free hemoglobin. Atherosclerosis 2007, 191(1):48-53.

14. Quaye IK: Haptoglobin, inflammation and disease. Trans $R$ Soc Trop Med Hyg 2008, 102(8):735-742.

15. Levy AP: Haptoglobin: a major susceptibility gene for diabetic cardiovascular disease. Isr Med Assoc J 2004, 6(5):308-310.

16. Asleh R, Levy AP: In vivo and in vitro studies establishing haptoglobin as a major susceptibility gene for diabetic vascular disease. Vasc Health Risk Manag 2005, 1(1):19-28.

17. Spagnuolo MS, Cigliano L, D'Andrea LD, Pedone C, Abrescia P: Assignment of the binding site for haptoglobin on apolipoprotein A-I. J Biol Chem 2005, 280(2):1193-1198.

18. Watanabe J, Chou KJ, Liao JC, Miao Y, Meng HH, Ge H, Grijalva V, Hama S, Kozak K, Buga G, et al: Differential association of hemoglobin with proinflammatory high density lipoproteins in atherogenic/ hyperlipidemic mice. A novel biomarker of atherosclerosis. J Biol Chem 2007, 282(32):23698-23707.

19. Levy AP, Hochberg I, Jablonski K, Resnick HE, Lee ET, Best L, Howard BV: Haptoglobin phenotype is an independent risk factor for cardiovascular disease in individuals with diabetes: the strong heart study. J Am Coll Cardiol 2002, 40(11):1984-1990.

20. Suleiman M, Aronson D, Asleh R, Kapeliovich MR, Roguin A, Meisel SR, Shochat M, Sulieman A, Reisner SA, Markiewicz W, et al: Haptoglobin polymorphism predicts 30-day mortality and heart failure in patients with diabetes and acute myocardial infarction. Diabetes 2005, 54(9):2802-2806.
21. Simpson M, Snell-Bergeon JK, Kinney GL, Lache O, Miller-Lotan R, Anbinder Y, Rewers MJ, Levy AP: Haptoglobin genotype predicts development of coronary artery calcification in a prospective cohort of patients with type 1 diabetes. Cardiovasc Diabetol 2011, 10:99.

22. De Bacquer D, De Backer G, Langlois M, Delanghe J, Kesteloot H, Kornitzer M: Haptoglobin polymorphism as a risk factor for coronary heart disease mortality. Atherosclerosis 2001, 157(1):161-166.

23. Levy AP, Larson MG, Corey D, Lotan R, Vita JA, Benjamin EJ: Haptoglobin phenotype and prevalent coronary heart disease in the Framingham offspring cohort. Atherosclerosis 2004, 172(2):361-365.

24. Stern MP, Ferrell RE, Rosenthal M, Haffner SM, Hazuda HP: Association between NIDDM, RH blood group, and haptoglobin phenotype. Results from the San Antonio heart study. Diabetes 1986, 35(4):387-391.

25. Quaye IK, Ababio G, Amoah AG: Haptoglobin 2-2 phenotype is a risk factor for type 2 diabetes in Ghana. J Atheroscler Thromb 2006, 13(2):90-94.

26. Bowden DW, Lehtinen AB, Ziegler JT, Rudock ME, Xu J, Wagenknecht LE, Herrington DM, Rich SS, Freedman Bl, Carr JJ, et al: Genetic epidemiology of subclinical cardiovascular disease in the diabetes heart study. Ann Hum Genet 2008, 72(Pt 5):598-610.

27. Carr JJ, Nelson JC, Wong ND, McNitt-Gray M, Arad Y, Jacobs DR Jr, Sidney S, Bild DE, Williams OD, Detrano RC: Calcified coronary artery plaque measurement with cardiac $\mathrm{CT}$ in population-based studies: standardized protocol of multi-ethnic study of atherosclerosis (MESA) and coronary artery risk development in young adults (CARDIA) study. Radiology 2005, 234(1):35-43.

28. Carr JJ, Crouse JR 3rd, Goff DC Jr, D’Agostino RB Jr, Peterson NP, Burke GL: Evaluation of subsecond gated helical $C T$ for quantification of coronary artery calcium and comparison with electron beam CT. AJR Am J Roentgenol 2000, 174(4):915-921.

29. Lange LA, Bowden DW, Langefeld CD, Wagenknecht LE, Carr JJ, Rich SS, Riley WA, Freedman BI: Heritability of carotid artery intima-medial thickness in type 2 diabetes. Stroke 2002, 33(7):1876-1881.

30. Koch W, Latz W, Eichinger M, Roguin A, Levy AP, Schomig A, Kastrati A: Genotyping of the common haptoglobin $\mathrm{Hp} 1 / 2$ polymorphism based on PCR. Clin Chem 2002, 48(9):1377-1382.

31. Bento JL, Palmer ND, Zhong M, Roh B, Lewis JP. Wing MR, Pandya H, Freedman Bl, Langefeld CD, Rich SS, et al: Heterogeneity in gene loci associated with type 2 diabetes on human chromosome 20q13.1. Genomics 2008, 92(4):226-234.

32. Lewis JP, Palmer ND, Ellington JB, Divers J, Ng MC, Lu L, Langefeld CD, Freedman BI, Bowden DW: Analysis of candidate genes on chromosome 20q12-13.1 reveals evidence for BMI mediated association of PREX1 with type 2 diabetes in European Americans. Genomics 2010, 96(4):211-219.

33. Matarin M, Brown WM, Scholz S, Simon-Sanchez J, Fung HC, Hernandez D, Gibbs JR, De Vrieze FW, Crews C, Britton A, et al: A genome-wide genotyping study in patients with ischaemic stroke: initial analysis and data release. Lancet Neurol 2007, 6(5):414-420.

34. Grant DJ, Maeda N: A base substitution in the promoter associated with the human haptoglobin 2-1 modified phenotype decreases transcriptional activity and responsiveness to interleukin-6 in human hepatoma cells. Am J Hum Genet 1993, 52(5):974-980.

35. Maeda N: DNA polymorphisms in the controlling region of the human haptoglobin genes: a molecular explanation for the haptoglobin 2-1 modified phenotype. Am J Hum Genet 1991, 49(1):158-166

36. Cox SE, Doherty C, Atkinson SH, Nweneka CV, Fulford AJ, Ghattas H, Rockett KA Kwiatkowski DP, Prentice AM: Haplotype association between haptoglobin (Hp2) and Hp promoter SNP (A-61C) may explain previous controversy of haptoglobin and malaria protection. PLoS One 2007, 2(4):e362.

37. Froguel P, Ndiaye NC, Bonnefond A, Bouatia-Naji N, Dechaume A, Siest G, Herbeth B, Falchi M, Bottolo L, Gueant-Rodriguez RM, et al: A genome-wide association study identifies rs2000999 as a strong genetic determinant of circulating haptoglobin levels. PLoS One 2012, 7(3):e32327.

38. Hamalainen P, Saltevo J, Kautiainen H, Mantyselka P, Vanhala M: Erythropoietin, ferritin, haptoglobin, hemoglobin and transferrin receptor in metabolic syndrome: a case control study. Cardiovasc Diabetol 2012, 11:116

39. Wobeto VP, Pinho Pda C, Souza JR, Zaccariotto TR, Zonati Mde F: Haptoglobin genotypes and refractory hypertension in type 2 diabetes mellitus patients. Ara Bras Cardiol 2011, 97(4):338-345. 
40. Ryndel M, Behre CJ, Brohall G, Prahl U, Schmidt C, Bergstrom G, Fagerberg $\mathrm{B}$, Olson FJ: The haptoglobin 2-2 genotype is associated with carotid atherosclerosis in 64-year old women with established diabetes. Clin Chim Acta 2010, 411(7-8):500-504.

41. Nakagawa T, Muramoto Y, Hori M, Mihara S, Marubayashi T, Nakagawa K: A preliminary investigation of the association between haptoglobin polymorphism, serum ferritin concentration and fatty liver disease. Clin Chim Acta 2008, 398(1-2):34-38.

doi:10.1186/1475-2840-12-31

Cite this article as: Adams et al: Genetic analysis of haptoglobin polymorphisms with cardiovascular disease and type 2 diabetes in the diabetes heart study. Cardiovascular Diabetology 2013 12:31.

\section{Submit your next manuscript to BioMed Central and take full advantage of:}

- Convenient online submission

- Thorough peer review

- No space constraints or color figure charges

- Immediate publication on acceptance

- Inclusion in PubMed, CAS, Scopus and Google Scholar

- Research which is freely available for redistribution 